\title{
Aerodynamic streams at cylindrical internal grinding by the textured wheels
}

\author{
Vladimir Gusev ${ }^{1, *}$ \\ ${ }^{1}$ Institute of Engineering and Automobile Transport,Vladimir State University named under \\ Alexander and Nikolay Stoletovs, Russia
}

\begin{abstract}
During internal grinding a large amount of heat is formed. A heat has a negative impact on all processing indicators. The speed of heat removal from the processed workpiece is defined not only by structure of grinding wheel and by giving method of lubricant cooling liquid (LCL), but also by the aerodynamic streams, which are formed by a tool rotation. Aerodynamics of traditional grinding wheels is studied in detail, but for textured wheels to aerodynamic streams did not pay of due attention. The multiple-factor experiment is executed and models of movement speed of the aerodynamic streams are determined. It is established, that the greatest influence on the movement speed of the air flows has an axial distance of a measurement point from an end face of abrasive segments and a radial distance of this point from the cutting surface. Static pressure of air in an internal wheel's cavity is equal 47 Pas, and outside of the wheel in close proximity to the cutting surface -212 Pas. Taking into account the received experimental data of the movement speeds of aerodynamic streams and different data of air pressure in the specified areas, possible methods of giving of LCL in a cutting zone are analysed. It is established, that the most effective is the centrifugal method of giving of LCL to an internal cavity of the textured tool.
\end{abstract}

\section{Introduction}

At grinding a large amount of heat, having negative impact practically on all of process indicators, is formed. Therefore a heat removal from a cutting zone with a high speed, represents a scientific problem. Speed of heat removal depends from a grinding wheel construction, giving method of LCL on the processed surface, and other factors. For elimination of a negative action of powerful aerodynamic streams on a movement of LCL to a cutting zone was offered a method of an internal grinding in the closed cavity, filled by LCL [1]. Unrotative grinding wheel with radially mobile abrasive segments is situated into the cavity, where the workpiece is fixed. The cavity is closed by cover and filled LCL. Include work movements of a wheel and workpiece and carry out draft, semi-fair, fair and finishing processing of workpiece, at reducing of a wheel rotation frequency. Separating of the aerodynamic streams, generated by a grinding wheel, from a cutting zone, dispersal

\footnotetext{
* Corresponding author: prof_gusev@mail.ru
} 
elimination of LCL by the rotating abrasive segments will allow to increase a heat removal from a workpiece at internal grinding [2]. In works [3-6] on the basis of a research of air flows distribution was provided the guaranteed presence of LCL in a cutting zone. Have been created the LCL curtains, that fence a perimeter of cutting zone and eliminate spraying of a liquid. Aerodynamic streams are investigated also in works [7-10], on the basis of which the effective giving way of LCL is offered.

In works $[11,12]$ supply of compressed air through vortex tubes is investigated. The intensive district stream, axial layers of which are considerably cooled and go away through a diaphragm in the form of the cold stream, directed to a cutting zone, is formed. At the present stage of development of grinding processes much attention is paid to a research of the so-called textured abrasive and diamond grinding wheels [13-16], which open new effective ways of an intensification of heat removal by interruption of cutting process and creation powerful aero - and hydrodynamic streams. Insufficient scientific information about distribution of aerodynamic streams and values of their movement speeds at grinding by textured wheels leads to the unreasonable choice of a LCL giving to a cutting zone and to decreasing in efficiency of grinding processes.

\section{Technique of experimental study of aerodynamic streams}

The textured wheel for cylindrical internal grinding of holes represents a metal case 1 (Fig. 1) with longitudinal grooves, into them the cutting abrasive segments 2 , divided among themselves by air intervals, are inserted.

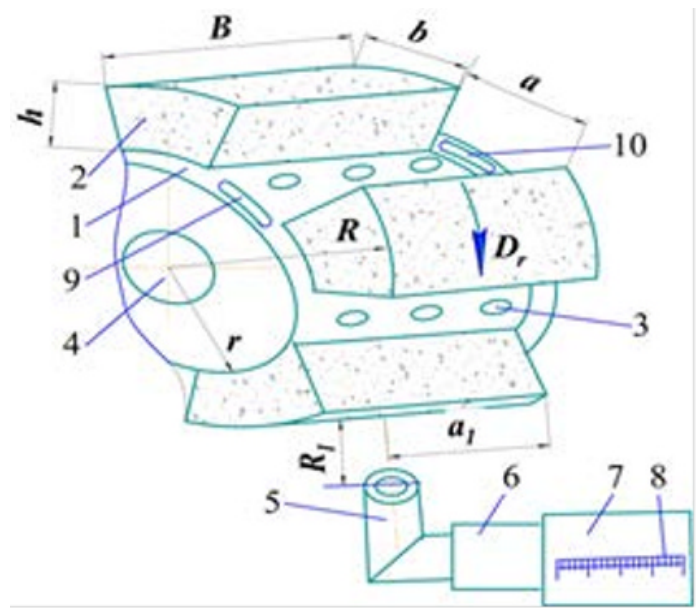

Fig. 1. The measurement scheme of the speed of aerodynamic stream, generated by the textured grinding wheel.

The length $h$ of used part of abrasive segment is equal:

$$
h=R-(r+1),
$$

where $\mathrm{R}$ - is the radius of the cutting surface of the textured grinding wheel; $r$ - radius of an external cylindrical surface of the case 1 .

Height of abrasive segment 2 is equal $B$, and its extent in the district direction is equal $b$. In case 1 radial holes 3 for giving of LCL in a cutting zone are made. The textured wheel is established by the central hole 4 on a spindle of the grinding machine tool and in the course 
of grinding rotates with high frequency on an arrow $D_{r}$, generating a powerful aerodynamic streams. The speed of the stream movement was measured by means of a Pitot tube 5, connected by a rubber tube 6 to micro manometer 7 of MMN-1 model, of a scale 8, established at an angle to the horizontal plane. For definition of multiple-factor model of air streams speed are executed the experiences according to a matrix of the central composite orthogonal planning of the second order.

Are chosen the independent varied factors: a distance $a_{1}$ (code $x_{1}$ ) from the right end face of abrasive segments to Pitot's tube; distance $R_{1}$ ( code $x_{2}$ ) of Pitot's tube from the cutting surface; length $h$ (code $x_{3}$ ) of the wear part of a segment; the distance $a$ (code $x_{4}$ ) between adjacent segments. Values of independent factors are presented in the table 1.

Table 1. Values of independent factors

\begin{tabular}{|c|c|c|c|c|}
\hline Factors & $a_{1} \mathbf{m m}$ & $R_{1}, \mathbf{m m}$ & $h, \mathbf{m m}$ & $a, \mathbf{m m}$ \\
\hline Code & $x_{1}$ & $x_{2}$ & $x_{3}$ & $x_{4}$ \\
\hline Main level & 10 & 7.5 & 6.25 & 12.5 \\
\hline Variation interval & 7 & 4.6 & 2.65 & 5.3 \\
\hline Top level (+1) & 17 & 12.1 & 8.90 & 17.8 \\
\hline Lower level (-1) & 3 & 2.9 & 3.60 & 7.2 \\
\hline Star point $(+\alpha=+1.414)$ & 20 & 14.0 & 10.0 & 20.0 \\
\hline Star point $(-\alpha=-1.414)$ & 0 & I.0 & 2.50 & 5.0 \\
\hline Zero point & 10 & 7.5 & 6.25 & 12.5 \\
\hline
\end{tabular}

A priori the postulated mathematical model, connecting the speed $V$ (code $Y$ ) of aerodynamic streams with the specified factors, was found in the form of a polynomial:

$$
\begin{aligned}
& Y=b_{0}+b_{1} x_{1}+b_{2} x_{2}+b_{3} x_{3}+b_{4} x_{4}+b_{12} x_{1} x_{2}+b_{13} x_{1} x_{3}+b_{14} x_{1} x_{4}+ \\
& +b_{23} x_{2} x_{3}+b_{24} x_{2} x_{4}+b_{34} x_{3} x_{4}+b_{11} x_{1}^{2}+b_{22} x_{2}^{2}+b_{33} x_{3}^{2}+b_{44} x_{4}^{2}
\end{aligned}
$$

where $b_{0} b_{1}-b_{4}$-are the regression coefficients; $x_{1}-x_{4}$-kodes of independent factors.

\section{Multiple-factor models of the movement speed of district aerodynamic stream}

After realization of a planning matrix, statistical processing of experiment results the regression equation (1), which is checked for adequacy with use of Fischer-criterion, is defined. As a result of the executed procedures the multiple-factor model of the movement speed of district aerodynamic stream is received:

$$
\begin{array}{r}
V=-27.5+5.4 a_{1}-5.1 R_{1}+7.4 h+1.5 a+0.18 R_{1} h- \\
-0.1 a_{1} a-0.18 h a-0.18 a_{1}^{2}+0.2 R_{1}^{2}-0.5 h^{2} .
\end{array}
$$


The analysis (3) shows, that the speed of an aerodynamic stream significantly depends on factors $a_{1}$. Dependences are described by the full trinomials, containing a factor in the second, first and zero degree and represented by parabolas with various arrangement of top and branches. Tops of parabolas are located on a direct, parallel to an ordinates axis, and are remote from the coordinates start at the distance, equal to a half of height $B$ of abrasive segment (Fig. 2, a).

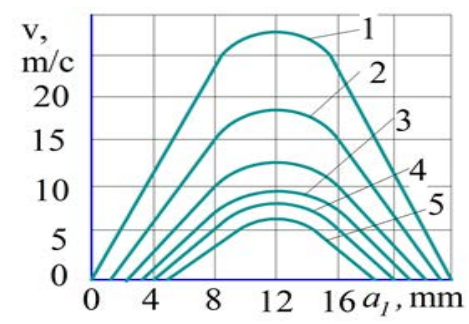

a

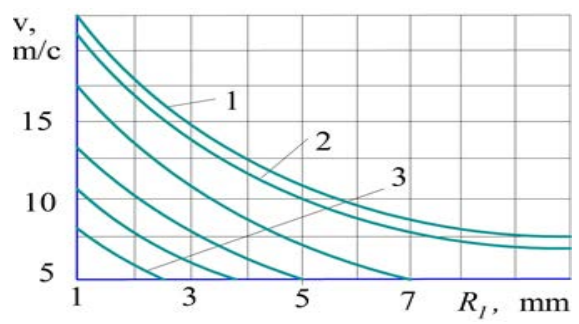

b

Fig. 2. Change of speed of an aerodynamic district stream as length forming of a segment (a) and as distance of a measurement point.

Curves $4-8$ are described respectively by the equations:

1. $V=-0.2+4.15 a_{1}-0.18 a_{1}^{2}$, at $R_{1}=1 \mathrm{~mm}, h=6.25 \mathrm{~mm}, a=12.5 \mathrm{~mm}$;

2. $V=-6.11+4.15 a_{1}-0.18 a_{1}^{2}$, at $R_{1}=3 \mathrm{~mm}$, same values of $h$ and $a$;

3. $V=-14.0+4.15 a_{1}-0.18 a_{1}^{2}$, at $R_{1}=7 \mathrm{~mm}$, same values of $h$ and $a$;

4. $V=-15.4+4.15 a_{1}-0.18 a_{1}^{2}$, at $R_{1}=9 \mathrm{~mm}$, same values of $h$ and $a$;

5. $V=-15.5+4.15 a_{1}-0.18 a_{1}^{2}$, at $R_{1}=11 \mathrm{~mm}$, same values of $h$ and $a$.

With increase in distance $R_{1}$ speed $V$ quickly decreases (Fig. 2, b), what is explained by brake of an aerodynamic district stream by the air surrounding. Curves 9-11 are described respectively by the equations:

$$
V=25.7-4.2 R_{1}+0.2 R_{1}^{2} \text {, at } a_{1}=11 \mathrm{~mm}, h=5 \mathrm{~mm}, a=17.8 \mathrm{~mm} \text {; }
$$

2. $V=24.0-4.0 R_{1}+0.2 R_{1}^{2}$, at $a_{1}=12 \mathrm{~mm}, h=6.25 \mathrm{~mm}, a=17.2 \mathrm{~mm}$;

3. $V=12.2-3.5 R_{1}+0.2 R_{1}^{2}$, at $a_{1}=10 \mathrm{~mm}, h=9 \mathrm{~mm}, a=20 \mathrm{~mm}$.

Height $h$ of abrasive segments and an air interval $a$ have considerably less impact on the speed of an aerodynamic stream, than factors $a_{1}$ and $R_{1}$. 


\section{The movement speed of end face aerodynamic streams}

Thus, the maximum values of speeds of the aerodynamic streams, generated by the textured wheel, are observed in the area, located close to the cutting surface and in the middle of abrasive segments. On the basis of this fact it is possible to assume, that a direction of LCL to the cutting surface will not provide effective action of coolant, because its penetration into a cutting zone will be followed by a considerable resistance of aerodynamic streams. The end face aerodynamic streams, generated by the textured wheel, are much weaker than of a district stream and almost do not depend on a measurement point location concerning an axis of rotation of the tool (Fig. 3 , a).

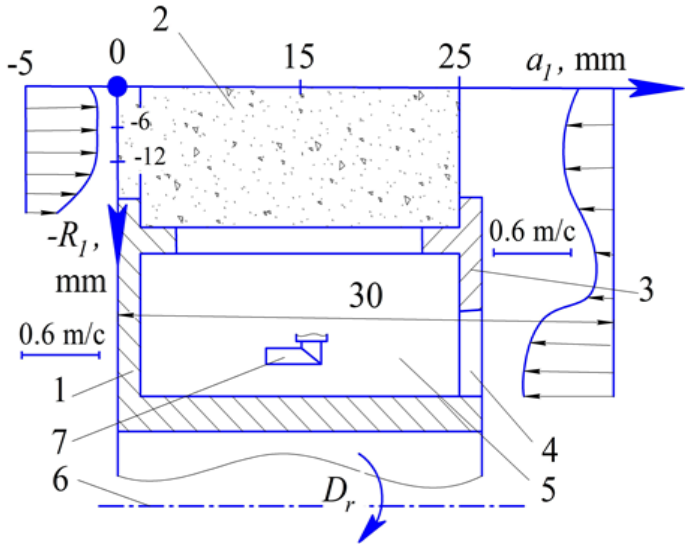

a

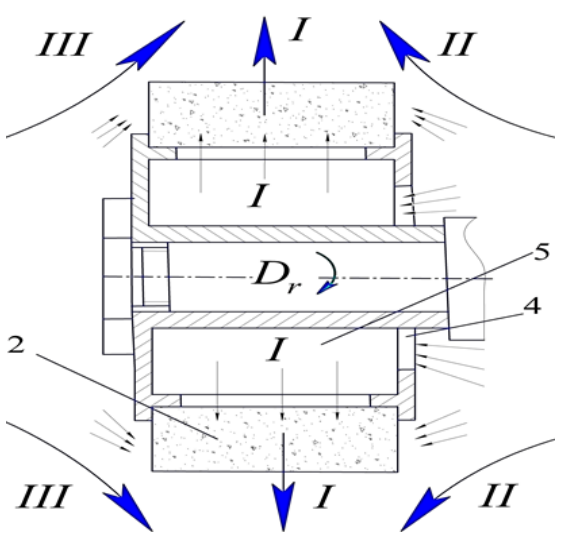

b

Fig. 3. The and face aerodynamic streams (a), generated by a wheel: 1 - the case, 2 - an abrasive segment, 3 - a cover, 4 - a ring gap, 5 - an internal cavity, 6 - a rotation axis of a wheel; 7 - Pitot's tube; a trajectory of the aerodynamic streams (b).

Static pressure of air in the tool (Pitot's tube 7 is established in a cavity 5) is equal 47 Pas, and outside in a cross plane of symmetry of abrasive segments, in close proximity to the cutting surface - 212 Pas, what testifies to essential pressure difference in the specified areas. This fact should be taken in attention at choosing a way of giving of a cooling to a cutting zone. At wheel rotation on an arrow $D_{r}$ the aerodynamic flow $I$ passes through a ring gap 4 into a cavity 5 (Fig. 3, a) and under influence of centrifugal force through a pores of abrasive segments 2 and radial holes 3 (Fig. 1) - to the cutting zone and processed surface. Flows $I I$ and $I I I$ arrive respectively from the right and left end face of abrasive segments. On exit of wheel the flows I, II and III are unite with each other and form the powerful aerodynamic stream, surrounding the cutting surface of a grinding wheel. Flows I-III will promote passing of the LCL to a cutting zone, if the directions of the movement of these streams and LCL coincide, but in this case two devices for giving of $L C L$ are required. The devices are situated in close proximity of end faces, what complicates an installation and adjustment of devices. Besides, there is a difficulty with providing a pressure, equal on all height of segment. For the specified reasons a giving of CLC in the direction of flows II and III is unsatisfactory. In view of the small static pressure of air (47 Pas) in an internal cavity 5 (Fig. 3, a) it is possible to expect, that the giving of LCL in the direction of an aerodynamic flow $I$ will be the best. In this case an uniform distribution of CLC on all height of a segment can be provided by a direction of CLC through district grooves 9 and 10 (Fig. 1). 
Thus, at grinding by the textured wheel the best effect is provided by a coolant giving in a direction of an aerodynamic flow $I$ into the grinding wheel, and from there - to a cutting zone through radial holes 3, and the district grooves 9 and 10 (Fig. 1). In this case the grinding wheel will be separated from flows $I I$ and $I I I$ by the streams of LCL, which follow from the grooves 9 and 10. The provisions, formulated above, are checked experimentally. On a spindle of the grinding machine tool $3 \mathrm{~A} 227 \mathrm{~V}$ was established the textured grinding wheel of next sizes: external diameter (diameter of the cutting surface) $-D=63 \mathrm{~mm}$, a segment's height $B=20 \mathrm{~mm}$, a central hole's diameter $-20 \mathrm{~mm}$. A quantity of abrasive segments in the grinding wheel -6 pieces.

A marking of abrasive material of segments: 24AF90L6V5. Coolant was given by different ways: in the form of one and several streams, directed radially to the tool, an opposite to a cutting zone; in the form of two streams, directed parallel to an axis of wheel, near a cutting zone; in the form of the conic stream, sent into a reception cavity of a wheel, through a ring gap 4 (Fig. 3, b). The current of LCL in a cutting zone was estimated visually by using of a stroboscopic tachometer. Experiments showed, that the best results on compactness and distribution of LCL in a cutting zone are provided by a giving through a ring gap 4 in a cavity 5 of the tool, in the same time the other ways were characterized by intensive spraying, dispersion of LCL and by its current outside of a cutting zone.

Thus, a research of the aerodynamic streams, generated by the textured grinding wheel, allows to choose reasonably the most effective way of LCL giving at round internal grinding, what will have a positive impact on the quality indicators of processed details.

\section{Conclusion}

1. The aerodynamic streams, accompanying processes of grinding, have a great influence on the current and action efficiency of a lubricant cooling liquid in a cutting zone and are deeply studied for traditional grinding by wheels with the continuous cutting surface. In relation to the textured grinding wheels due attention was not paid to such researches in spite of the fact, that they open new effective ways of an intensification of heat removal from a cutting zone.

2. The multiple-factor experiment is planned, the experiences of a planning matrix are executed, statistical processing of experiment results is made, and the models, which connect the speeds of aerodynamic streams with the independent factors, characterizing the sizes of the textured tool and the coordinates of measurement points of air masses speeds, are received.

3. On the basis of the established experimental models the speeds of aerodynamic streams and of static air pressure in and outside of the textured grinding wheel are determined. On the basis of the obtained scientific information, possible ways of coolant giving in a cutting zone are worked out. Analysis of these ways allowed to choose the most effective centrifugal way of coolant's giving at grinding by the textured wheels.

\section{References}

1. Patent 2182531. RU, (2002)

2. D. R. Blurtsyan. Centrifugal Internal Grinding by Assembled Wheel with Radially

Mobile Segments. Mat. Sci. Forum. Advances in Abrasive Technology XIX, Vol. 874.

Trans Tech Publ., Switzerland, (2016) 
3. Yu. V. Vasilenko, The Current state of the technology of giving LCL when grinding. Chief mech. Eng, RU. Moscow. No 2, (2008)

4. Yu. V. Vasilenko, A. V. Tyukhta, The Combinatory way of giving LCL at flat grinding by the periphery of a wheel. RU. Samara. Vol. 13. No4(4), (2011)

5. A.V. Tyukhta, Mathematical model of interaction of a particle of slime with a curtain from LCL at flat grinding by a periphery of a wheel. Fundamental and applied problems of the equipment and technology. No3-2 (293), (2012)

6. A. V. Tyuhta, Combinatorial method for cooling lubricating fluid supply at flat grinding by periphery of wheel. The 13-th Joint Chi-RU sym. on advanced materials and processing technology. Articles of int. sci. tech. conf., (2012)

7. V.G. Gusev, P.S. Shvagirev, A.V. Morozov, The Aerodynamic streams, generated by a discrete face grinding wheel. Int. sci. tech. conf. RU. Tula, (2004)

8. L.V. Khudobin, etc. The lubricant cooling technological means and their application when processing by cutting: Reference book. RU. Moscow, (2006)

9. D. S. Rechenko, E. S., Rechenko, M. A. Walther, Research of high-speed grinding. Modern problems of mech. Eng. The VI-th Int. sci. tech. conf., (2008)

10. Z. I. Kremen, V. G. Yuryev, A. F. Baboshkin, Technology of grinding in mechanical engineering. RU. SPb, (2007)

11. A. M. Dolganov, Increase in efficiency of grinding due to use of the tool with vortex cooling. Int. sci. tech. conf. RU. Volggasu, (2006)

12. A. M. Dolganov, The Perspective grinding tool with vortex cooling. All-RU sci. tech. pr. conf. Novosibirsk, (2006)

13. H. N. Li and D. Axinte, Textured grinding wheels: A review, Int. J. of Machine Tools and Manufacture, (2016)

14. M. J. Jackson, M. P. Hitchiner, High performance grinding and advanced cutting tools, Springer Verlag, (2012)

15. E. Oliveira, C. Silva, F. G. Hashimoto, Industrial challenges in grinding, CIRP Annals Manuf. Tech. 58 (2), (2009) 